\title{
高铁发展对城市对间联系的影响及异质性研究 以南京市为例
}

\author{
刘梦雨, 沈丽珍“
}

(南京大学建筑与城市规划学院, 南京 210093)

\begin{abstract}
摘 要: 快速发展的高铁网络对区域社会经济产生了巨大影响,而高铁发展对具有不同属性的城市对间联系的影响 机制仍亟需探讨。论文选取南京市 2000-2019年全行业的企业对外横向投资联系数据,测度以南京市为核心的 城市间经济联系变化,在揭示城市对间联系格局的基础上,定量识别和分析了高铁因素对不同属性城市对影响的 异质性。研究表明: (1) 南京市与全国不同城市间的联系强度呈现出明显的层级性、区域性分布和门户性特征; (2) 城市对间的经济水平差异和交通联系强度是城市对间联系强度的关键影响因素, 高铁的发展加强了城市对间的联 系强度, 在一定程度上加剧了城市间联系地理格局的极化现象; (3) 高铁因素对不同类型的城市对产生的影响程度 具有明显的异质性,时间距离短、政策指导合作度高、较大 GDP 差异、较小创新能力差异的城市对类型更容易受益 于高铁的发展。根据研究结论, 可以对具体城市参与城市网络建构、点对点发展城市间联系进行一定的干预和导 向,在高铁的影响下进一步有指向性地带动城市间要素流动。
\end{abstract}

关 键 词:高铁; 城市联系; 城市网络; 流动空间;城市对; 南京

中国高铁的快速发展增强了全国范围内城市 的可达性, 产生了明显的“时空收玫” 和“走廊效 应” ${ }^{[1-2]}$ 。在目前高铁网络全覆盖的国家政策规划背 景下, 高铁作为一种属于政府政策干预的城市基础 设施, 旨在通过高铁网络的介人来改善区域经济发 展差异。研究显示, 中国的高铁网络显著地促进了 区域一体化, 大幅度提升了沿线城市的可达性, 为 许多城市提供了区位优势, 而高铁对可达性和连通 性产生的影响会不可避免地对经济活动的迁移、集 聚和扩散产生长期影响 ${ }^{[3-5]}$ 。

目前, 高铁网络对中国社会经济影响的研究主 要集中在经济、经济地理、城市地理、城市网络等 领域, 尤其以经济领域居多。研究内容大多聚焦 在 3 个方面: (1) 高铁发展对城市网络和交通网络 的影响, 主要通过各种可达性和连通性指标进行 度量 ${ }^{[1,2,6-10]}$; (2) 高铁发展对城市及区域经济的影响, 主要包括对区域经济差异、经济活动再分配、区域
一体化、产业集聚和专业化以及经济投资等方面的 影响 ${ }^{[3,5,11-21]}$; (3) 高铁发展对其他交通方式的影响,特 别是对航空经济的影响 ${ }^{[8,15,22-23]}$ 。在研究层面上, 大 量研究以各大区域、省域为研究对象, 特别是对城 市群的研究较多; 针对某条线路沿线城市的研究 中, 以东南部线路居多,对于中西部来说主要是探 讨某条线路对于省域内城市和经济的影响。近来 也有部分学者关注到了高铁建设对于城市间经济 联系的影响, 大多从旅游视角出发, 使用旅游接待 人次、人口规模等面数据构建引力模型来表征城市 旅游经济联系, 通过定性观察高铁开通前后旅游经 济联系的变化,证实了高铁对不同区域的城市间旅 游经济联系存在异质性影响 ${ }^{[2-29]}$ 。

但目前高铁对区域经济、城市间联系影响的研 究存在以下不足:(1) 高铁对城市间联系影响的研究 多集中在旅游网络上, 亟需从全局的角度把握高铁 对城市经济联系的影响; (2) 面数据和静态数据对城

收稿日期:2020-07-14; 修订日期:2020-10-15。

基金项目: 国家自然科学基金项目(41871160)。[Foundation: National Natural Science Foundation of China, No. 41871160. ]

第一作者简介:刘梦雨(1996-),女,河北唐山人,硕士生,研究方向为城市与区域规划。E-mail: liumy_33@163.com

*通信作者简介: 沈丽珍(1976-), 女,福建三明人,博士,副教授,研究方向为城市与区域规划。E-mail: shellyjun@163.com

引用格式: 刘梦雨, 沈丽珍. 高铁发展对城市对间联系的影响及异质性研究: 以南京市为例 [J]. 地理科学进展, 2021, 40(4): 647-659. [Liu Mengyu, Shen Lizhen. Impact of high-speed railway network on inter-city connections and its heterogeneity: Taking Nanjing City as the center of network. Progress in Geography, 2021, 40(4): 647-659. ] DOI: 10.18306/dlkxjz.2021.04.009 
际联系的动态性考虑不足, 且引力模型构建的城市 网络存在一些局限性, 如用动态的流数据能更好地 表征城市间联系; (3) 定性分析或简单的相关性检验 虽探讨了高铁的某些影响机制, 但不能排除其他因 素对城市发展及城际联系变化的干扰, 如时空距 离、政策指导、经济发展差异等; 特别是在异质性方 面, 很少有研究明确指出高铁对城市间联系的具体 影响差异。

流动空间的视角与高铁的要素携带特征较为 契合, 作为一种动态的变化过程, 高铁对于两两特 定的城市对间联系的具体影响差异如何? 作为一 种载体, 高铁同时承载了实体流与虚拟流, 显著缩 短了商业旅行时间, 由此假设, 公司间的合作或公 司内部的母子联系可能加强, 从而增强了城市间的 经济流动,重构城市网络体系。

因此, 本文从流动空间的视角出发, 使用动态 流数据, 即全行业的企业横向投资联系数据表征两 两城市对之间的联系强度, 通过对一定数量的城市 对样本进行回归, 并模拟无高铁的情况下城市对联 系格局, 将高铁因素独立出来后定量探究其对具有 不同属性的城市对间联系的影响机制和异质性, 依 托流数据和定量分析对相关研究进行补充, 并根据 其影响异质性提供点对点城市联系发展建议。

\section{1 研究区域、数据与方法}

\section{1 研究地理单元选取}

南京市作为长三角城市群中唯一的特大城市, 占据良好的区位优势和创新资源, 但近年来与长三 角其他大城市相比, 存在城市发展动力不足、人口 迁移活跃度明显下降等问题 ${ }^{[30]}$ 。自 2010 年 7 月南 京市第一条高铁沪宁城际通车、2011年第一座高铁 站南京南站随京沪高铁开通投人使用, 至目前已通 达24个省会城市,规划中的南京北站和“米”字形高 铁路网均已在建, 将极大增强南京市与沿线城市间 的交通联系。可见南京市不仅是中国城市发展的 一个典型样本, 也是全国铁路综合交通枢纽, 具有 一定的代表性。因此选择南京市为中心城市, 通过 表征以南京市为起论点的要素流人与流出情况、南 京市与全国其他城市的联系强度变化, 探究在当今 高铁网络所引起的交通联系变化对不同类型城市 对之间的影响异质性。

已有研究所选取的地理单元多为以省域内城
市或特定线路的沿线城市为主，一定程度上忽视了 城市间跨省域自发形成的联系、受到其他线路过境 影响而产生的辐射作用等。因此, 为避免主观性影 响, 本文根据数据呈现结果, 选取与南京市产生企 业间横向联系的地级市及以上级别城市(不包括港 澳台地区)为地理研究单元。

\section{2 研究方法与数据来源}

\subsection{1 研究方法}

(1) 城市网络分析方法

信息化、全球化背景下,不同企业之间广泛的 合作与投资产生了大量的横向联系数据, 相对传统 的 “总部一分支”企业数据, 这些横向投资联系数据 更能够反映城市间的网络连接关系 ${ }^{[3]}$ 。因此, 部分 学者开始探究如何利用企业间的联系数据构建城 市网络, 如 Pan 等 ${ }^{[32]}$ 提出了利用APS(advanced producer service)公司在首次公开募股的数据、李哲睿 等 ${ }^{[33]}$ 选取全行业的海量投资数据进行了相关研究。

借鉴已有研究, 本文以某城市中的企业对异地 城市企业投资频次之和表示出度, 以某城市中的企 业被异地城市企业投资频次之和代表人度, 出度与 人度之和即为两两城市对间的联系强度值 $\left(C_{i j}\right)$ 。此 处的投资频次不包括相同企业内部“总部一分支” 的投资结构, 以期与传统连锁网络模型的数据选取 原则相区别。考虑高速铁路影响效果的滞后性, 选 取2010、2014、2019年3 个时间断面进行比较。此 外,有别于一般城市网络研究中“多对多”的网络状 联系格局,为聚焦城市对类型差异而避免不同城市 差异的复杂性, 将南京作为参照城市, 选取“一对 多” 的放射状联系进行城市对的研究, 以探究高铁 对城市联系的具体影响机制。

\section{(2) 建立回归方程}

本文利用相关性检验和回归分析, 探究两两城 市对间联系强度 $\left(C_{i j}\right)$ 的影响因素并构建回归方程, 以便进行后续控制变量的研究。城市间的联系受 到复杂多元因素的共同影响,根据本文的主要研究 目的并借鉴已有研究, 从空间、经济、交通和行政 4 个方面选取如下 9 个指标作为初步解释变量(表 1), 后续经过相关性检测笁选变量构建回归模型。

1) 地理距离 $\left(x_{11}\right)$ 。空间距离远近可能会影响城 市间的联系强度。以两城市行政中心的欧氏距离 表征地理距离。

2) 时间距离 $\left(x_{12}\right)$ 。两城市间的时间距离会影响 到城市对间的沟通效率。以两城市间的铁路加权 
最短旅行时间计算时间距离,计算公式为:

$$
T_{i j}=w_{\mathrm{h}} \cdot t_{\min 1}+w_{\mathrm{n}} \cdot t_{\min 2}
$$

式中: $T_{i j}$ 表示两城市间的时间距离, $w_{\mathrm{h}}$ 表示两城市 之间高铁动车班次 $(\mathrm{C} / \mathrm{G} / \mathrm{D}$ 字头列车 $)$ 占铁路总班次 的比重, $t_{\min 1}$ 表示高铁动车所用最短旅行时间; $w_{\mathrm{n}}$ 表 示普通列车班次占铁路总班次的比重, $t_{\min 2}$ 表示普 通列车所用最短旅行时间。

3) 城市 GDP 差异 $\left(x_{21}\right)$ 。反映城市的总体经济 状况, 许多研究表明城市的经济发展与城市对外联 系具有相关性。

4) 城市资金总量之差 $\left(x_{22}\right)$ 。反映城市汇聚金融 资金的能力, 可能会对吸引投资能力有一定影响。

5) 人均可支配收人之差 $\left(x_{23}\right)$ 。代表了两城市居 民的收人差距, 潜在地影响城市间人口的流动。

6) 行业关系 $\left(x_{24}\right)$ : 通过 Krugman 结构差异度指 数表征城市对的产业结构关系:

$$
\mathrm{KI}_{i j}=\sum_{k=1}^{n}\left|X_{i k}-X_{j k}\right|
$$

式中: $\mathrm{KI}_{i j}$ 是结构差异度指数, $X_{i k}$ 是城市 $i$ 的 $k$ 产业占 整个产业的比重, $X_{j k}$ 是城市 $j$ 的 $k$ 产业占整个产业的 比重。 $\mathrm{KI}_{i j}$ 的值介于 $0 \sim 2, \mathrm{KI}_{i j}$ 越接近于 0 则两城市间 的产业同构程度越高, 反之同构程度越低。

7) 基于铁路网的交通联系。交通流中的高铁 联系是本文试图研究的主要因素。借鉴已有研究交 通网络和交通流的指标 ${ }^{[34-35]}$, 分别以城市间往返高铁 班次数 (高铁联系 $x_{31}$ ) 和普通列车班次数 (普铁联系 $x_{32}$ )分别表征城市对间基于铁路网的交通流强度。

$8)$ 行政关系 $\left(X_{4}\right)$ 。中国具有典型的 “行政区经 济现象”, 省级政府是制定和落实政策的单位; 且有 研究显示城市网络的社区结构受地域和省界影响 较大 ${ }^{[8]}$ 。因此将同在江苏省的城市对赋值为 1 , 否则 为 0 。

\section{(3) 控制变量模拟}

根据所得回归模型, 控制其他因素不变, 模拟 高铁开通情况与 2010 年无差别的城市对间联系强 度值, 通过以下算式判断相同变化值的高铁联系对 不同城市对的影响程度 $(E)$ :

$$
\begin{gathered}
E=\left(\Delta C_{i j}-\Delta C_{i j}{ }^{\prime}\right) /\left(H_{i j}^{2019}-H_{i j}^{2010}\right) \\
\Delta C_{i j}=C_{i j}^{2019}-C_{i j}^{2010} \\
\Delta C_{i j}{ }^{\prime}=C^{201 j}{ }^{2019}-C_{i j}^{2010}
\end{gathered}
$$

式中: $\Delta C_{i j}$ 为 2019 年与 2010 年实际城市联系值的 差值; $\Delta C_{i j}{ }^{\prime}$ 为 2019 年模拟联系值与 2010 年实际城
表 1 城市对间联系强度回归方程的解释变量选取

Tab.1 Explanatory variables of the regression equation for the strength of city pair connections

\begin{tabular}{cl}
\hline 一级指标 & \multicolumn{1}{c}{ 二级指标 } \\
\hline 空间 $\left(X_{1}\right)$ & 地理距离 $\left(x_{11}\right)$ \\
& 时间距离 $\left(x_{12}\right)$ \\
经济 $\left(X_{2}\right)$ & GDP差异 $\left(x_{21}\right)$ \\
& 城市资金总量之差 $\left(x_{22}\right)$ \\
& 人均可支配收人之差 $\left(x_{23}\right)$ \\
& 行业关系 $\left(x_{24}\right)$ \\
& 高铁联系 $\left(x_{31}\right)$ \\
交通 $\left(X_{3}\right)$ & 普铁联系 $\left(x_{32}\right)$ \\
& 行政关系 $\left(x_{4}\right)$ \\
行政 $\left(X_{4}\right)$ &
\end{tabular}

市联系值的差值; $H_{i j}^{2019}$ 为 2019 年高铁班次数目, $H_{i j}^{2010}$ 为 2010 年高铁班次数目; $C_{i j}^{2019}$ 为 2019 年实际 的城市对间联系值; $C_{i j}^{2010}$ 为 2010 年实际的城市对 间联系值; $C^{\prime 2019}$ 为 2019 年模拟高铁无发展情况下 的城市对间联系值。

$\Delta C_{i j}$ 与 $\Delta C_{i j}{ }^{\prime}$ 之差即高铁网络联系一直处于 2010 年水平、控制其他因素影响不变的情况下, 城 市对间联系变化值与实际变化值之差。

$E$ 为 $\Delta C_{i j}$ 与 $\Delta C_{i j}{ }^{\prime}$ 之差和 $H_{i j}^{2019}$ 与 $H_{i j}^{2010}$ 之差的 比值, 可避免受到不同城市间高铁班次变化量多少 的影响, 即 $E$ 为高铁联系变化单位值导致的城市对 联系值变化, 以此表征高铁因素的影响强度。

最后, 以城市对的属性指标作为解释变量, 通过 相关性分析和回归分析解释在同样的高铁发展条件 下, 具有何种属性的城市对受到的影响程度更大、 更容易从高铁联系的加强中获益。

(4) 空间自相关分析

空间自相关分析可以解决高铁因素对不同城 市对的影响特征是否存在空间集聚性、相关性的问 题, 以免忽视空间特征导致分析的不合理性。最常 用的全局空间自相关衡量指标是 Moran's $I$ 指数, 其 值在 $-1 \sim 1$ 之间, 大于 0 表示存在空间正相关,小于 0 表示存在空间负相关, 等于 0 表示随机分布。

\subsection{2 数据来源}

自行编写 Python 程序, 从 “百度信誉” 网站 (https://xin.baidu.com) 抓取相关企业数据和投资信 息。“百度信誉”为商业调查网站,数据来源主要为 国家企业信用信息公示系统。选取经营地址为南 京市的全行业企业,保留注册资金在 100 万元以上、 
营业状态在业的企业, 爬取该企业列表所有股东企 业和对外投资企业及其所在城市, 剔除南京市内部 的投资联系, 仅保留跨城市横向投资数据, 最终得 到有效数据 5248 条, 以此作为基础数据表征城市间 联系强度。2019年城市对间高铁和普铁的相关数 据来自于 12306 官方网站, 2010 年的铁路相关数据 来自于石开旅行时刻表 2010 版; 回归和模拟分析过 程所需要的各类城市属性数据均来自于各城市政 府的统计公报; 专利数据来源于中国专利公布公告 网站(http://epub.sipo.gov.cn/)。

\section{2 城市间联系格局及变化分析}

\section{1 南京市对外联系特征}

根据 2019年断面联系数据, 可以得到南京市与 不同城市之间的联系格局。

(1) 联系强度值的层级式分布。

为避免企业投资选择的偶然性,选取 2019年联 系强度值在 8 以上的城市, 利用 ArcGIS 中的自然断 裂点分级法将其分为 6 个等级 (表 2 ), 可以看出, 城 市间联系强度呈现明显的层级式分布和极化现 象。联系强度较高的前 3 个层级的城市数量远远小
于第四层级的城市数量。第一层级的上海作为长 三角区域网络中的核心城市，与南京市之间的联系 强度明显高于其他城市; 北京是全国的政治经济文 化中心, 联系强度也远超其他城市。第二层级的深 圳、苏州均为重要的区域性节点城市。

(2) 城市区域性分布和跨区域的“门户性”。

对不同层级的城市的地理属性进行分类,发现 与南京市联系的城市具有明显的区域聚集性分布 特征(图 1)。其中与南京市同处于长江三角洲城市 群的城市上海、苏州、杭州、无锡和宁波等与南京市 联系强度值较强, 第四、五、六层级中的城市有 41.8\%都集中在长三角区域。部分长三角区域外的 高等级城市与南京市之间的联系强度明显低于区 域内次一级城市的联系强度,表明高等级城市之间 联系未必强于次级城市间的联系,这也体现了目前 城市等级体系正在逐渐向城市网络转变。

而跨区域的联系具有明显的核心性,或称之为 “门户性”。各个层级联系总值较高的跨区域城市 均为其所在区域的门户型城市。一般来说,门户型 城市是企业在不同阶段进行空间扩展首先选择的 区位 ${ }^{[36]}$,与门户型城市的联系对于加强与其所在区 域的联系具有重要作用。例如,北京、天津是京津

表2 2010-2019年南京市对外联系强度变化

Tab.2 Comparison of changes of Nanjing's external connections from 2010 to 2019

\begin{tabular}{|c|c|c|c|}
\hline \multirow{2}{*}{ 层级 } & \multicolumn{3}{|c|}{ 城市(与南京市联系强度) } \\
\hline & 2010年 & 2014年 & 2019年 \\
\hline 层级一 & 北京(105)、上海(100) & 上海(349)、北京(325) & 上海(933)北京(727) \\
\hline 层级二 & 深圳(31)、苏州(27) & 深圳(148)、苏州(126) & 深圳(416)、苏州(316) \\
\hline 层级三 & $\begin{array}{l}\text { 无锡(22)、烟台(20)、淮安(18)、徐 } \\
\text { 州(17)、乌鲁木齐(16)、宁波(15)、 } \\
\text { 扬州(15) }\end{array}$ & $\begin{array}{l}\text { 无锡(76)、杭州(64)、镇江(51) 宁波(48)、扬州 } \\
(48) \text { 、广州 (46)、淮安 (43)、徐州 (42)、泰州 } \\
\text { (40)、天津 (38)、常州 (38)、合肥 (37)、南通 } \\
\text { (36)、盐城(35) }\end{array}$ & $\begin{array}{l}\text { 杭州 (189)、无锡 (172)、宁波 (142)、镇江 } \\
\text { (119)、徐州(115)、扬州(110) }\end{array}$ \\
\hline 层级四 & $\begin{array}{l}\text { 合肥(13)、天津(13)、杭州(13)、泰 } \\
\text { 州(13)、广州(12)、常州(11)、盐城 } \\
\text { (11)、西安(9)、镇江(9) }\end{array}$ & $\begin{array}{l}\text { 滁州 (25)、烟台 (23)、西安 (21)、乌鲁木齐 } \\
(20) \text { 、重庆(19)、厦门(18)、济南(18)、马鞍山 } \\
\text { (16)、连云港(16)、宿迁(16)、青岛(16)、武汉 } \\
\text { (15)、大连(14)、长沙(14)、拉萨(14)、成都(27) }\end{array}$ & $\begin{array}{l}\text { 淮安(88)、合肥(87)、广州(82)、天津(81)、常 } \\
\text { 州(77)、南通(76)、盐城(70)、泰州(63)、拉萨 } \\
\text { (56)、成都(54) }\end{array}$ \\
\hline 层级五 & $\begin{array}{l}\text { 南通(8)、青岛(7)、武汉(7)、滁州 } \\
(7) 、 \text { 长沙(6)、济南(5)、成都(5)、海 } \\
\text { 口(4)、德州(4)、哈密(4)、大连(4)、 } \\
\text { 马鞍山(4) }\end{array}$ & 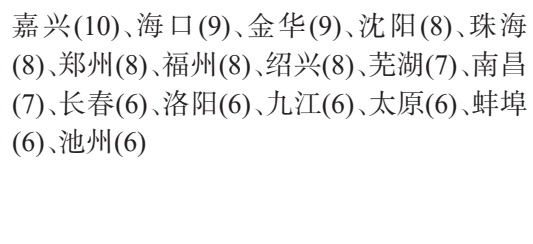 & $\begin{array}{l}\text { 青岛(47)、厦门(44)、滁州(44)、宿迁(42)、重 } \\
\text { 庆(42)、连云港(39)、西安(39)、嘉兴(39)、武 } \\
\text { 汉(38)、济南(31)、马鞍山(30)、乌鲁木齐 } \\
\text { (30)、烟台(29)、长沙(27)、湖州(24)、珠海 } \\
\text { (24)、南昌(23)、郑州(22)、芜湖(21)、九江 } \\
\text { (21)、福州(21)、金华(20)、大连(18) }\end{array}$ \\
\hline 层级六 & $\begin{array}{l}\text { 宿迁(3)、厦门(3)、连云港(3)、长春 } \\
\text { (3)、南昌(3)、福州(3)、洛阳(2)、九 } \\
\text { 江(2)、昌吉(2)、淄博(2)、淮北(2)、 } \\
\text { 临沂(2)、嘉兴(2)、鹰潭 (2)、沈阳 } \\
\text { (2)、十堰(2)、威海(2)、台州(2)、巴 } \\
\text { 中(2)、东莞(2)、绍兴(2) }\end{array}$ & 临沂(5)、哈密(5)、湖州(5)、佛山(5)、台州(5) & $\begin{array}{l}\text { 伊犁(17)、绍兴(16)、沈阳(15)、海口(13)、 } \\
\text { 太原(12)、东莞(11)、新余(11)、宣城(11)、 } \\
\text { 蚌埠(10)、济宁(9)、哈尔滨(9)、营口(9)、昆 } \\
\text { 明(9)、台州(9)、温州(9)、贵阳(9)、长春(8)、 } \\
\text { 德州(8)、日照(8) }\end{array}$ \\
\hline
\end{tabular}




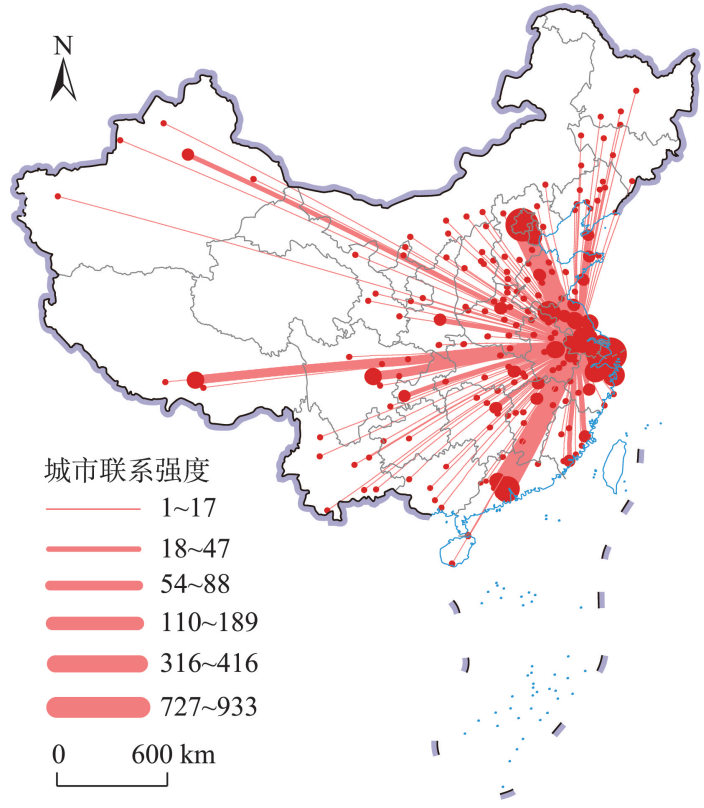

注:本图基于自然资源部标准地图服务网站下载的审图 号为 GS(2019)1697号的标准地图制作, 底图无修改,下同。

图 1 2019年南京市对外联系地理分布

Fig.1 Geographical distribution of connections of Nanjing City, 2019

冀城市群的门户城市, 深圳、广州等是珠三角城市 群的核心城市, 成都、重庆是成渝城市群的核心城 市。这些城市在南京市的对外联系强度排名中均 排位较高。

\section{2 南京市对外联系格局演化特征}

将2010、2014年的断面数据分别选取联系强度 值在 $2 、 5$ 以上的城市, 通过自然断裂点分级法将其 分为 6 个等级,并与 2019 年的数据进行对比(表 2), 发现:

(1) 极化现象加重。随着时间发展, 北京、上 海、深圳、苏州 4 个城市一直居于联系总值的前 4 位, 与南京市的联系不断加强, 特别是上海和北京 与南京市的联系强度远超其他城市; 前 3 层级城市 的数量逐渐减少, 更多的城市处于联系较弱的五、 六层级。

(2) 第三、四、五层级的城市组成发生了较大变 化, 从 2010 年发展至 2019 年, 最初长三角城市群区 域内城市联系占绝对优势, 而现在更多的跨区域核 心城市联系显著增强,例如广州、成都、天津、厦门 和拉萨。这表明随着社会经济和交通基础设施的 发展,特别是快速交通廊道的建设对城市的连接, 更多的城市不再局限于区域内部, 开始寻求跨区域 的合作。这一点根据城市联系的地理分布图(图 2)
也可得出。

\section{3 城市对间联系强度变化与高铁数量变化对比}

为尽量避免企业投资选择的偶然性,选取 2019 年断面城市联系强度值在 5 以上的共 74 组城市对 作为样本进行分析。

在各方面因素的影响下, $10 \mathrm{a}$ 间南京市与其他 城市之间的联系强度在量值和地理分布上都发生 了较大变化。为初步探究城市对间联系强度变化 和高铁数量变化之间的情况是否存在分异,将样本 城市对在 2010-2019年间两者变化情况的地理分 布图进行直观对比, 其中变化程度数据均进行标准 化处理, 以方便进行比较。

如图 3 所示,两者均具有典型的空间集聚性特 征,特别是城市对间高铁数量变化的集聚性特征更 加明显。此外,两者间存在一定的分异情况, 即高 铁联系数量提升程度高的城市对, 其城市间联系加 强程度却较低。因此,为探究高铁因素对何种属性 的城市对联系变化影响最大,在保证其他影响因素 不变的情况下,对高铁因素进行单独探讨。

\section{3 高铁因素的影响异质性分析}

\section{1 城市对间联系强度影响因素的回归方程建立}

针对表 1 中的变量指标, 利用统计软件 SAS 的 CORR 过程进行相关性检验, 以对解释变量进行初 步篮选,结果显示(表 3): (1) 地理距离、时间距离均 与联系强度不相关,这表明在信息化、网络化的背 景下, 中心地理论弱化, 空间距离和时间距离受到 了不同程度的压缩, 城市之间的合作不再受到地 理、时间距离的限制, 与南京市建立联系的城市不 具有明显的空间集聚性,空间因素相对来说影响不 大; (2) 经济变量组中 GDP 差异、城市资金总量之差 和行业关系均具有显著相关性, 由此假设,城市对 间联系强度受到城市的总体经济水平、融资能力和 产业结构的影响; (3) 交通变量组的 2 个变量均具有 显著相关性,表明城市对间不同类型的铁路联系可 能都会对城市联系情况产生影响; (4. 行政关系因素 不相关,表明城市间联系不再局限于行政领域限 制,开始注重跨区域的合作。

经过相关性检测, 篮选出 GDP 差异 $\left(x_{21}\right)$ 、城市 资金总量之差 $\left(x_{22}\right)$ 、行业关系 $\left(x_{24}\right)$ 、高铁联系 $\left(x_{31}\right)$ 、普 铁联系 $\left(x_{32}\right) 5$ 个变量, 利用 SAS 软件的 REG 过程进 行回归拟合, 采用 Mallow's Cp 法选择回归模型, 经 
(a) 2010年

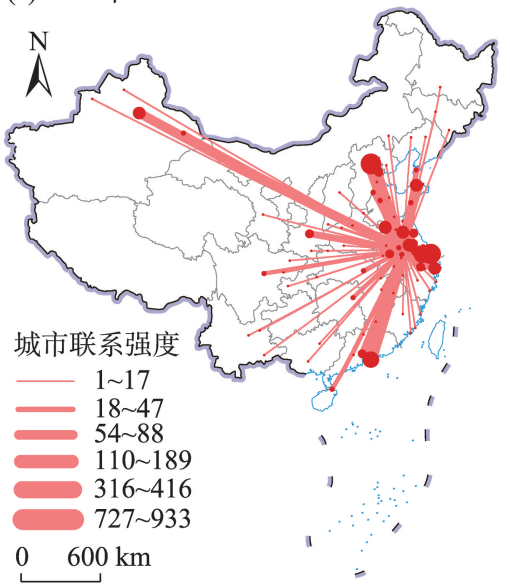

(b) 2014年

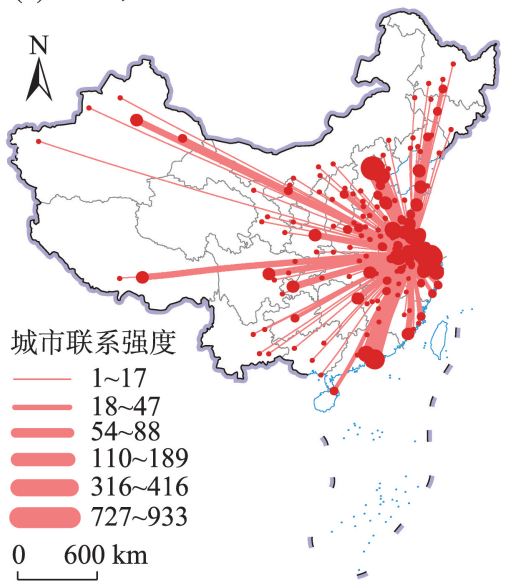

(c) 2019 年

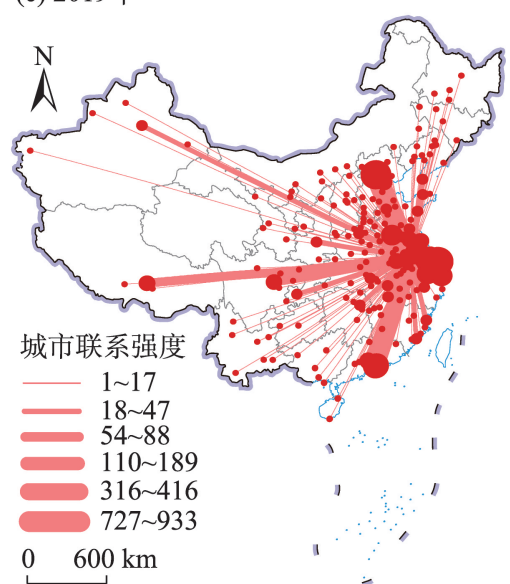

图2 2010、2014、2019年南京市对外联系地理格局变化

Fig.2 Comparison of changes in the geographical pattern of Nanjing's external connections in 2010, 2014 and 2019

(a) 城市联系变化程度

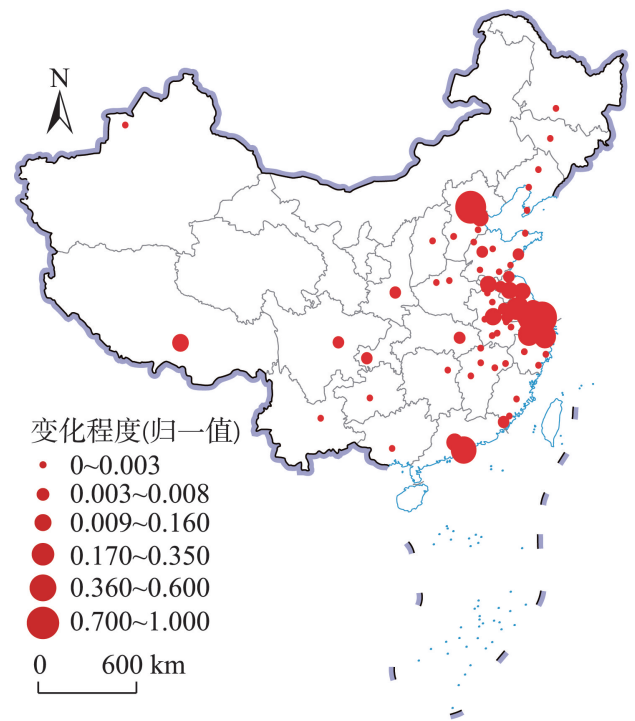

(b) 高铁联系变化程度

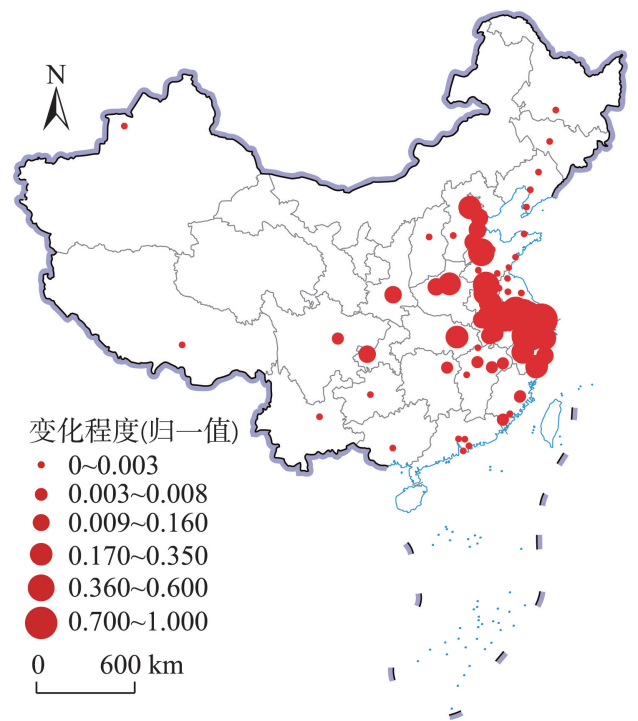

图 32010 -2019年各城市与南京市间联系强度变化及高铁联系变化地理分布

Fig.3 Change in the strength of connections between other cities and Nanjing and the geographical distribution of the change in high-speed rail connections from 2010 to 2019

过添加高次项和变量多次调整, 最终得到回归结果 如表 4 所示, 调整 $R^{2}$ 为 0.9528 , 说明回归方程拟合程 度较好。解释变量的 $t$ 检验显示, 只有普铁联系对 方程的解释效果较差;共线性诊断结果显示不同类 的解释变量之间相互独立, 因此认为变量设置较为 合理。

表 4 的回归结果表明: (1) 城市间联系的影响因 素复杂多样且并非简单的线性相关,相关性检验中 高度相关的各变量对城市间联系的影响或存在较 大差距; 2 城市对间的经济距离是影响城市间联系 的重要因素; 3 交通联系中高铁的影响比普铁的影
响明显更强,其原因是由于高铁的发展大大压缩了 时空距离,而普铁并未显著提高交通联系效率, 且 近 $10 \mathrm{a}$ 来普铁的修建发展缓慢。

最后得到回归方程如下：

$$
\begin{aligned}
C_{i j}= & \alpha+\beta_{1} x_{21}+\beta_{2}\left(x_{21}\right)^{2}+\beta_{3}\left(x_{21}\right)^{3}+\beta_{4} x_{22}+ \\
& \beta_{5}\left(x_{22}\right)^{2}+\beta_{6}\left(x_{22}\right)^{3}+\beta_{7}\left(x_{24}\right)^{3}+\beta_{8}\left(x_{31}\right)^{2}+ \\
& \beta_{9}\left(x_{32}\right)^{3}+\varepsilon
\end{aligned}
$$

式中: $\alpha$ 为常数项, $\beta_{1} 、 \beta_{2} \cdots \cdots \beta_{9}$ 为各变量的回归系 数, $\varepsilon$ 为残差项。

利用上述回归方程对高铁水平与 2010 年相同、 
表 3 SAS 系统 CORR 过程各因素相关性检验结果

Tab.3 SAS system CORR process correlation test results

\begin{tabular}{clc}
\hline 一级指标 & \multicolumn{1}{c}{ 二级指标 } & $P$ 值 \\
\hline 空间 $\left(X_{1}\right)$ & 地理距离 $\left(x_{11}\right)$ & -0.0707 \\
& 时间距离 $\left(x_{12}\right)$ & -0.1165 \\
经济 $\left(X_{2}\right)$ & GDP差异 $\left(x_{21}\right)$ & $0.7008^{* * *}$ \\
& 城市资金总量之差 $\left(x_{22}\right)$ & $-0.3157^{* *}$ \\
& 人均可支配收人之差 $\left(x_{23}\right)$ & 0.0848 \\
& 行业关系 $\left(x_{24}\right)$ & $0.4108^{* * *}$ \\
交通 $\left(X_{3}\right)$ & 高铁联系 $\left(x_{31}\right)$ & $0.5767^{* * *}$ \\
& 普铁联系 $\left(x_{32}\right)$ & $0.4033^{* * *}$ \\
行政 $\left(X_{4}\right)$ & 行政关系 $\left(x_{4}\right)$ & 0.1006 \\
\hline
\end{tabular}

注: ****和 $* * *$ 分别表示通过 $0.05 、 0.01 、 0.001$ 显著性水平检 验。下同。

其他变量不变情况下的城市对联系强度进行模拟， 并根据式(3)进行高铁影响程度的计算和分析。

\section{2 高铁对城市联系影响的异质性分析}

\subsection{1 高铁因素影响下的城市对联系格局对比}

控制其他变量不变, 将 2010 年高铁联系数值代 人回归方程(6)模拟 2019 年高铁无发展情况下的城 市对间联系强度值 $C_{i j}^{2019}$, 分析高铁因素单独产生 的影响。如图 4 所示, 南京市对外的实际联系格局 以京津、长三角城市群和广州深圳为 3 大核心, 呈现 出明显的轴带状; 而在高铁无发展的模拟联系格局 中, 城市对间联系强度整体下降,但极化现象有所 减弱, 出现了 3 大区域核心和许多门户型城市次级 核心, 整体呈现放射状格局; 且实际与模拟中的城 市在联系强度等级分布发生较大变化。

由此推断, 高铁的发展不仅大幅加强了城市对
间的联系,并且在一定程度上加剧了城市间联系的 极化特征,且高铁因素对不同的城市对影响不同。

\section{2 .2 城市对属性指标选取}

冷炳荣等 ${ }^{[37}$ 将城市网络的形成机制从社会分 工、技术进步和地方背景 3 个方面进行组织。本文 借鉴其对城市网络的解释框架,选择以下指标衡量 不同城市对的属性,作为解释变量(表 5),探究其与 高铁对城市对联系的影响程度之间的关系：

(1) 空间距离 $\left(Y_{1}\right)$ 。相同单位值的高铁联系变 化可能会由于城市对间的空间距离远近而产生不 同的影响,包括地理距离 $\left(y_{11}\right)$ 和时间距离 $\left(y_{12}\right)$, 其计 算方法与 1.2 .1 一节中相同。

(2) 地方背景 $\left(Y_{2}\right)$ 。经济行为和文化行为的密 切关系使得城市社会分工过程中有地方文化要素 嵌人 ${ }^{[38]}$, 不同的社会阶层对文化的需求存在差异, 而社会分层则造成了社会流动主体的差异,从而导 致了对不同产业对外联系的影响 ${ }^{[37}$ 。在现有研究 中有学者采用方言指标表征文化邻近性 ${ }^{[39-40]}$, 考虑 到地理和文化的复杂性, 本文根据方创琳等 ${ }^{[4]}$ 发布 的中国人文地理综合区划确定文化邻近性 $\left(y_{21}\right)$, 一 组城市对同在一个大区内的同一个二级区赋值为 2 , 同在一个地理大区内但不在同一个二级区赋值 为 1 , 不在一个地理大区内赋值为 0 。此外, 针对区 域合作、跨区域点对点合作等政策因素, 以在南京 政府网站搜索到的相关城市合作文件和信息公开 数量表征城市对间的行政合作关系 $\left(y_{22}\right)$ 。

(3) 社会分工 $\left(Y_{3}\right)$ 。信息技术和承载实体流的 基础设施网络的建设加速了企业生产的信息化和

表 4 SAS 系统 REG 过程多元非线性回归结果

Tab.4 SAS system REG process multiple nonlinear regression results

\begin{tabular}{lrrrc}
\hline \multicolumn{1}{c}{ 变量 } & 参数估计 & $t$ 值 & $P$ 值 & 方差膨胀因子 \\
\hline 截距 & 250.87398 & 9.69 & $<0.0001$ & 0 \\
$x_{21}$ & -183.29926 & -10.64 & $<0.0001$ & -2202 \\
$\left(x_{21}\right)^{2}$ & 62.55108 & 11.76 & $<0.0001$ & - \\
$\left(x_{21}\right)^{3}$ & -3.21477 & -11.08 & $<0.0001$ & 1.60951 \\
$x_{22}$ & -173.53229 & -4.34 & $<0.0001$ & - \\
$\left(x_{22}\right)^{2}$ & 99.90773 & 3.98 & 0.0002 & - \\
$\left(x_{22}\right)^{3}$ & -17.77985 & -3.92 & 0.0002 & 8.00454 \\
$\left(x_{24}\right)^{3}$ & -43.93322 & -5.68 & $<0.0001$ & 6.57463 \\
$\left(x_{31}\right)^{2}$ & 0.00123 & 3.82 & 0.0003 & 4.16759 \\
$\left(x_{32}\right)^{3}$ & $-1.727 \mathrm{E}-05$ & -0.67 & 0.5048 & \\
$R^{2}$ & 0.9586 & & & \\
调整 & 0.9528 & & & \\
\hline
\end{tabular}


(a) 实际联系格局

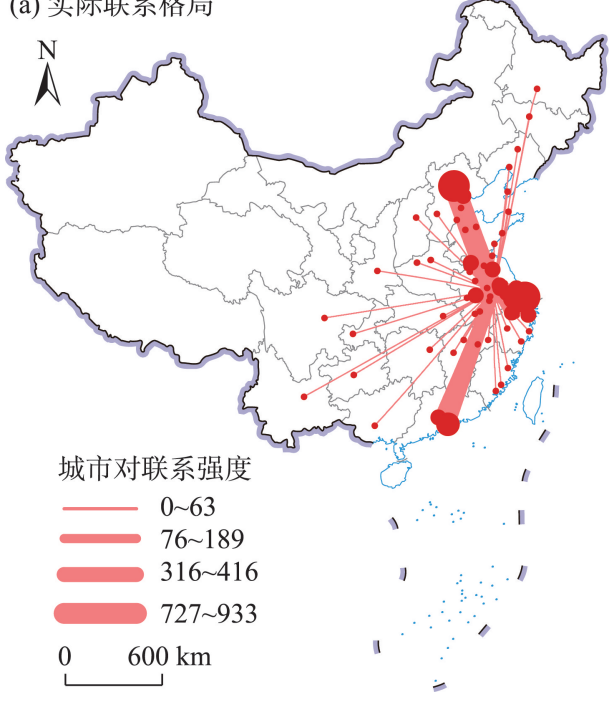

(b) 模拟联系格局

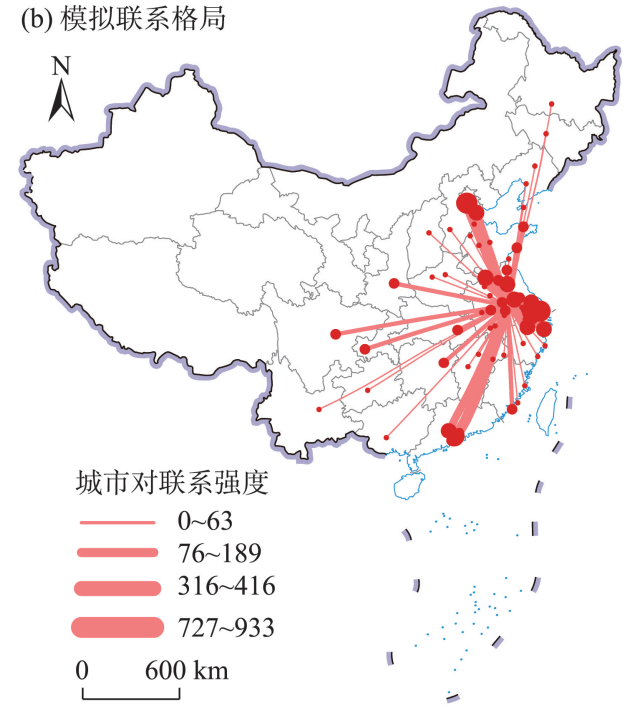

图 4 城市对样本实际联系格局与模拟联系格局对比

Fig.4 Comparison of actual and simulated connection patterns of city-dyad samples

表 5 城市对的属性指标选取

Tab.5 Selection of attributes of city-dyads

\begin{tabular}{cl}
\hline 一级属性指标 & \multicolumn{1}{c}{ 二级属性指标 } \\
\hline 空间距离 $\left(Y_{1}\right)$ & 地理距离 $\left(y_{11}\right)$ \\
& 时间距离 $\left(y_{12}\right)$ \\
地方背景 $\left(Y_{2}\right)$ & 文化邻近性 $\left(y_{21}\right)$ \\
& 行政合作关系 $\left(y_{22}\right)$ \\
& GDP 差异 $\left(y_{31}\right)$ \\
社会分工 $\left(Y_{3}\right)$ & 产业结构差异 $\left(y_{32}\right)$ \\
& 资金总量差异 $\left(y_{33}\right)$ \\
& 人均可支配收人差异 $\left(y_{34}\right)$ \\
& 对外发展特征 $\left(y_{41}\right)$ \\
技术进步 $\left(Y_{4}\right)$ & 创新能力差异 $\left(y_{42}\right)$ \\
&
\end{tabular}

空间分离, 高速铁路等基础设施为城市交流缩短了 空间交换成本, 加速产业重组 ${ }^{[37]}$, 从而导致了进一 步的社会分工和城市专业化。选取两城市间的 GDP 差作为整体经济发展差异 $\left(y_{31}\right)$; 另外, 由于服务 业易与其他产业和人口流动相关联, 同时也是产生 城市间联系的主体, 因此根据 Krugman 结构差异度 指数选择两城市间的产业结构差异作为行业关系 差异 $\left(y_{32}\right)$, 差异越小城市对的社会分工和产业组织 结构越相似; 此外,城市的资金总量差异 $\left(y_{33}\right)$ 体现了 城市的融资能力, 高铁作为资金流的载体, 对融资 能力差异不同的城市其影响可能存在异质性; 同 理, 城市居民人均可支配收人差异 $\left(y_{34}\right)$ 的不同可能 会在同等高铁因素的影响下形成不同程度的人员 流动, 从而体现出高铁因素影响的异质性。

(4) 技术进步 $\left(Y_{4}\right)$ 。城市的对外联系越强越容
易得到技术扩散，从而更有利于城市的发展。而高 铁作为一种交流方式的创新载体, 使得城市更容易 捕捉到外部发展的机会 ${ }^{[37]}$ 。考虑偏好依附理论, 将 城市对外部发展特征 $\left(y_{41}\right)$ 和城市对创新能力差异 $\left(y_{42}\right)$ 纳人城市对属性指标中, 其中对外发展特征以 两城市的实际利用外资额表示; 创新能力差异以两 城市的专利数量之差表示。

\section{2 .3 空间回归及结果分析}

将 $C_{i j}^{2019}$ 代人式(5)中, 根据式(3)计算得出高铁 联系变化单位值对所有城市对样本的影响程度, 进 行统计分析。在分析时,剔除了与南京间高铁班次 一直为零的城市, 最后共计 64 组城市对样本。利用 空间计量软件 GeoDa 对高铁的影响程度进行空间 自相关分析,结果如表 6 和图 5 所示, 高铁联系变化 的影响程度强弱具有空间集聚性, 即城市对属性中 的地理空间特征是起到作用的。这与前文中城市 对联系强度变化和高铁联系变化的地理分布集聚 性特征相吻合(图 3)。

对城市对样本分别采用经典线性回归模型和 空间误差模型进行回归分析,探究何种属性指标对 高铁影响程度具有显著性贡献。根据回归分析结 果(表 7), 高铁因素对特定属性的城市对即时间距离 短、政策合作密切、GDP 差异大和创新能力差异小 的城市对影响程度较高。

(1) 与空间自相关性相符,城市对间的时间距 离属性与高铁影响程度相关, 时间距离短的城市对 更容易因高铁的发展加强联系; 地理距离相关性不 
显著, 这可能是由于并非所有地理距离相近的城市 之间均具有较高的高铁连通性。此结果表明, 高铁 网络的发展造成的时空压缩使得城市间的时间距 离缩短, 即降低了交流的时间成本, 从而大大提升 了两城市间的交流效率, 所以城市对间的时间距离 越小, 高铁开通对其间联系强度提升的影响就越 明显。

(2) 具有政策指导合作的城市对更容易受到高 铁因素带来的影响, 且政策合作程度越高, 高铁的 影响程度越大。这充分说明了政府政策指向的重 要性。由此推断,政策因素使得城市对间原本的联 系强度较高, 而城市合作则更偏向于选择原本就具 有良好基础的城市, 因此高铁带来的交通联系强度 增强、交流方式多样化和便捷化均会给城市对间的 联系带来巨大影响。

(3) 高铁对 GDP 差异大的城市对间的联系更容 易产生较大影响, 特别是空间距离也较远的城市 对, 原本可能由于交通联系阻碍了协作, 但高铁的 开通和发展为这样的城市对提供了大量交流机会, 因此城市对间的联系强度提升幅度较大, 所表现出 的高铁影响程度也较高。此外,在社会分工变量组 中, 除GDP差异外, 其他因素相关性均不显著, 原因

表 6 高铁联系变化的影响程度空间自相关检测

Tab.6 Spatial autocorrelation detection of influence degree of high-speed rail connection change

\begin{tabular}{ccccc}
\hline Moran's $I$ & $P$ & $Z$ & $E(I)$ & 均值 \\
\hline 0.6949 & 0.0010 & 13.6301 & -0.0159 & 0.0966 \\
\hline
\end{tabular}

可能是由于: (1) 本文所选取的城市级别均为地级市 及以上城市。高铁发展导致经济活动重新分配,使 得城市网络趋向集中,高层经济活动、服务业趋向 于沿线核心城市, 土地和劳动力消耗经济活动则被 分散至沿线小城镇, 因此较为明显的社会分工和产 业结构差异大多存在于级别相差较大的城市对 间。(2)产业结构相似的城市之间容易产生横向合 作,结构相差较大的城市之间则倾向于纵向分工, 而高铁的开通对 2 种经济流动方式都有明显的影 响,因此无法判定产业结构差异不同的城市对受到 的高铁影响程度不同。

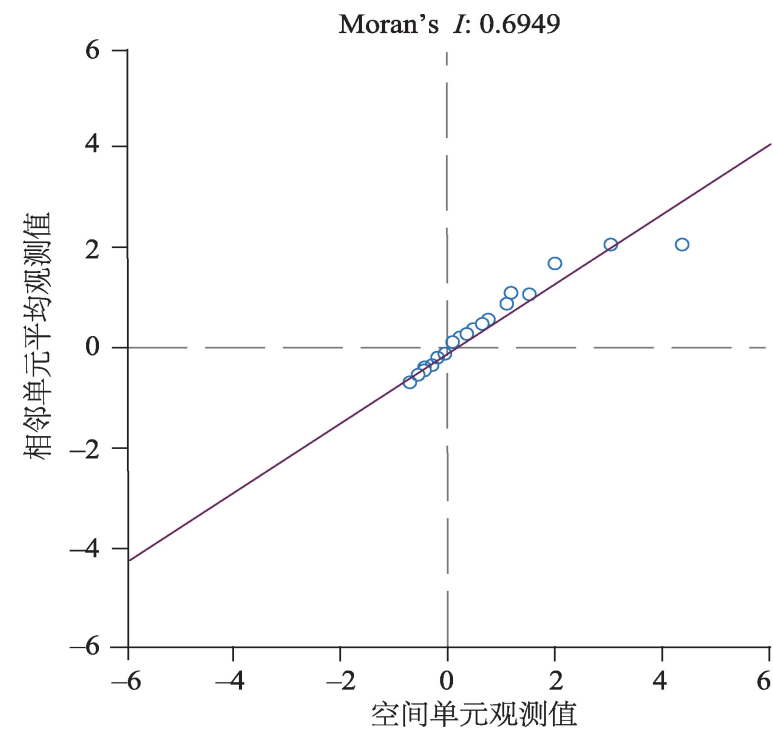

图 5 高铁联系变化的影响程度空间自相关检测

Fig.5 Spatial autocorrelation detection of influence degree of high-speed rail connection change

表 7 高铁因素影响程度的空间回归分析结果

Tab.7 Spatial regression analysis results of the degree of influence of high-speed rail

\begin{tabular}{llcc}
\hline & 变量 & 经典线性回归模型 & 空间误差模型 \\
\hline 空间距离 $\left(Y_{1}\right)$ & 地理距离 $\left(y_{11}\right)$ & $9.01 \mathrm{E}-05$ & 0.0001 \\
& 时间距离 $\left(y_{12}\right)$ & $-0.0191^{* *}$ & $-0.0196^{* * *}$ \\
地方背景 $\left(Y_{2}\right)$ & 文化邻近性 $\left(y_{21}\right)$ & 0.0213 & 0.0359 \\
& 行政合作关系 $\left(y_{22}\right)$ & $0.0018^{* *}$ & $0.0016^{* *}$ \\
社会分工 $\left(Y_{3}\right)$ & GDP 差异 $\left(y_{31}\right)$ & 0.0751 & $0.1096^{*}$ \\
& 产业结构差异 $\left(y_{32}\right)$ & -0.0573 & -0.0332 \\
& 资金总量差异 $\left(y_{33}\right)$ & -0.0054 & -0.0134 \\
& 人均可支配收人差异 $\left(y_{34}\right)$ & -0.0145 & -0.0176 \\
技术进步 $\left(Y_{4}\right)$ & 对外发展特征 $\left(y_{41}\right)$ & -0.0012 & -0.0010 \\
& 创新能力差异 $\left(y_{42}\right)$ & $-9.03 \mathrm{E}-07^{* *}$ & $-8.3148 \mathrm{E}-07^{* * *}$ \\
常数项 & & $0.3404^{* * *}$ & $0.2912^{* * * *}$ \\
$R^{2}$ & & 0.6412 & 0.6672 \\
空间残差项回归系数 & & & $0.2632^{*}$ \\
\hline
\end{tabular}


(4) 2 个城市创新能力差异越小, 高铁的发展越 能明显加强城市间的联系强度。技术进步变量组 的创新能力差异呈负相关, 对外发展特征相关性不 显著。这表明: (1) 城市的对外发展特征更多体现了 城市进出口经济水平, 对国内城市间交流贡献不 大; (2) 创新环境和能力则是一个城市发展的源动 力, 且这种城市对交通成本压缩和交流方式的创新 敏感性也较高, 2 个城市产生 “新工作” 的能力越接 近越容易产生联系, 需要面对面传输的“隐性知识” 也正是促进城市交流增加的优势, 而在前文提到高 铁正是因为能通过客流携带大量的“隐性知识”流， 其对创新能力集聚的城市对影响也就越强。

\section{4 结论与讨论}

\section{1 结论}

本文利用南京市全行业的横向投资联系构建 了以南京市为核心的全国城市要素流, 揭示了南京 市与其他城市间的联系格局及变化, 分析发现高铁 的发展大幅加强了城市对间的联系, 并且在一定程 度上加剧了城市间联系的极化特征, 对不同属性的 城市对影响存在明显的异质性。在流数据、提取高 铁因素定量模拟研究、两两城市对具体分析异质性 等方面对既有研究进行了一定程度的补充。具体 结论如下:

(1) 高铁因素对不同类型的城市对产生的影响 具有明显的异质性: 即高铁发展对时间距离短、政 策指导合作度高、较大 GDP 差异、较小创新能力差 异类型的城市对联系影响显著,在同等的高铁发展 水平下, 上述类型的城市对更容易受益于高铁, 明 显加强和提升联系强度;

(2) 经济水平差异和交通联系强度是城市对间 联系强度的关键影响因素, 在交通因素中高速铁路 的作用更加显著, 高铁的发展不仅加强城市对间的 联系强度, 并且在一定程度上加剧了城市间联系地 理格局的极化特征;

(3) 南京市与全国不同城市间的联系强度呈现 出明显的层级式分布、区域性分布和门户性特征。 在高铁发展和其他因素的影响下, 城市对间联系强 度的极化现象加重、跨区域联系加强, 其中上海、北 京、深圳和苏州一直占据前列, 长三角城市群内的 城市在数量上占有一定优势。

\section{2 讨论}

根据两两城市对相关研究结果, 可以对具体城
市参与城市网络建构进行一定的干预和导向, 特别 是针对某城市的点对点合作进行发展指导。面向 未来的全覆盖式、公交化的中国高铁规划, 为达到 通过建立区域快速交通廊道促进区域经济均衡发 展、城市更好地获益于高铁开通发展的目的, 针对 拥有高铁网络资源且有提升空间、试图加人城市网 络中、加强参与度、点对点提升联系强度的城市, 关 于未来发展提出以下建议:

(1) 抓住高铁网络化、与核心城市时间距离压 缩、交通流和人流上升带来的资源和机会, 积极应 对城市发展差距。如有意向加强城市间联系, 在高 铁通达条件较好的情况下, 应特别关注与目标城市 的政策合作和创新能力差异,应先从时间距离缩短 程度高、有政策指导基础、创新环境相似的网络节 点城市着手, 逐步增强自身的对外开放性,再从政 策方面积极谋求推动城市合作和投资,循序渐进寻 求与核心城市重点进行产业链承接, 参与区域产业 分工合作和专业化,以期增强城市间经济联系带动 发展。而在跨区域层面,双方政府的支持则显得尤 为重要,城市对间点对点的跨区域合作可能会为城 市发展提供全新的路径和动力。

(2) 拓宽城市的对外关系、大力提升城市创新 环境和能力。中小城市应当抓住高铁带来的会展、 会议和其他新交流机会,力争承接更多技术转移; 提升对小微企业和新技术企业的帮扶力度, 为创新 型人才提供良好的工作和居住环境, 只有创新型人 才和企业对外产生宽泛的联系,才能够带动城市间 的流动,形成正向反馈, 实现企业和城市发展的良 好互动, 从而更容易从高铁的发展中获益。

本研究的不足之处在于以南京市为核心, 从 “一对多”的要素流角度出发,对于区域内、跨区域 城市网络研究存在不足。因此,在未来的研究可对 扩展经济数据类型和城市对维度做进一步补充, 以 校验本文的研究结论。

\section{参考文献(References)}

[1] Cao J, Liu X C, Wang Y H, et al. Accessibility impacts of China's high-speed rail network [J]. Journal of Transport Geography, 2013, 28: 12-21.

[2] Shaw S L, Fang Z X, Lu S W, et al. Impacts of high speed rail on railroad network accessibility in China $[\mathrm{J}]$. Journal of Transport Geography, 2014, 40: 112-122.

[3] Chen C L. Reshaping Chinese space- economy through high-speed trains: Opportunities and challenges [J]. Journal of Transport Geography, 2012, 22: 312-316. 
[4] Ureña J M, Menerault P, Garmendia M. The high-speed rail challenge for big intermediate cities: A national, regional and local perspective [J]. Cities, 2009, 26(5): 266-279.

[5] Chen C L, Hall P. The impacts of high-speed trains on British economic geography: A study of the UK's InterCity 125/225 and its effects [J]. Journal of Transport Geography, 2011, 19(4): 689-704.

[6] Cheng Y S, Loo B P Y, Vickerman R. High-speed rail networks, economic integration and regional specialisation in China and Europe [J]. Travel Behaviour and Society, 2015, 2(1): 1-14.

[7] 赵丹, 张京祥. 高速铁路影响下的长三角城市群可达性 空间格局演变 [J]. 长江流域资源与环境, 2012, 21(4): 391-398. [Zhao Dan, Zhang Jingxiang. Research into spatial pattern changes of Yangtze River Delta's accessibility under the impact of high-speed railway. Resources and Environment in the Yangtze Basin, 2012, 21(4): 391-398. ]

[8] 王姣娥, 景悦. 中国城市网络等级结构特征及组织模式: 基于铁路和航空流的比较 [J]. 地理学报, 2017, 72(8): 1508-1519. [Wang Jiao'e, Jing Yue. Comparison of spatial structure and organization mode of inter- city networks from the perspective of railway and air passenger flow. Acta Geographica Sinica, 2017, 72(8): 1508-1519. ]

[9] 孙娜, 张梅青. 基于高铁流的中国城市网络结构特征演 变研究 [J]. 地理科学进展, 2020, 39(5): 727-737. [Sun $\mathrm{Na}$, Zhang Meiqing. Network structure and evolution characteristics of cities in China based on high-speed railway transport flow. Progress in Geography, 2020, 39(5): 727-737. ]

[10] 孙阳, 姚士谋, 张落成. 长三角城市群 “空间流”层级功 能结构: 基于高铁客运数据的分析 [J]. 地理科学进展, 2016, 35(11): 1381- 1387. [Sun Yang, Yao Shimou, Zhang Luocheng. Functional structure of spatial flow in the Yangtze River Delta: Analysis of passenger based data for the high speed railway. Progress in Geography, 2016, 35(11): 1381-1387. ]

[11] Yang H R, Dijst M, Witte P, et al. Comparing passenger flow and time schedule data to analyse high-speed railways and urban networks in China $[\mathrm{J}]$. Urban Studies, 2019, 56(6): 1267-1287.

[12] Li X J, Huang B, Li R R, et al. Exploring the impact of high speed railways on the spatial redistribution of economic activities: Yangtze River Delta urban agglomeration as a case study [J]. Journal of Transport Geography, 2016, 57: 194-206

[13] Sasaki K, Ohashi T, Ando A. High-speed rail transit impact on regional systems: Does the Shinkansen contribute to dispersion? [J]. The Annals of Regional Science,
1997, 31(1): 77-98.

[14] Chen Z H, Haynes K E. Impact of high-speed rail on regional economic disparity in China [J]. Journal of Transport Geography, 2017, 65: 80-91.

[15] (Ato) Xu W, Huang Y. The correlation between HSR construction and economic development: Empirical study of Chinese cities [J]. Transportation Research Part A: Policy and Practice, 2019, 126: 24-36.

[16] Tian M, Li T P, Yang S W, et al. The impact of high-speed rail on the service-sector agglomeration in China [J]. Sustainability, 2019, 11(7): 2128. doi: 10.3390/su11072128.

[17] 石林, 傅鹏, 李柳勇. 高铁促进区域经济一体化效应研 究 [J]. 上海经济研究, 2018, 30(1): 53-62, 83. [Shi Lin, Fu Peng, Li Liuyong. The effect of high-speed railway on regional economic integration. Shanghai Journal of Economics, 2018, 30(1): 53-62, 83. ]

[18] 李新光, 黄安民, 张永起. 高铁对区域经济发展的影响 评估: 基于 DID 模型对福建省的实证分析 [J]. 现代城 市研究, 2017, 32(4): 125-132. [Li Xinguang, Huang Anmin, Zhang Yongqi. An impact assessment of high-speed railway on regional economic development: An empirical analysis of Fujian Province based on DID model. Modern Urban Research, 2017, 32(4): 125-132. ]

[19] 市元超, 吴利华, 白俊红. 高铁开通、要素流动与区域 经济差距 [J]. 财贸经济, 2018, 39(6): 147-161. [Bian Yuanchao, Wu Lihua, Bai Junhong. High-speed rail, factor flow and regional economic disparities. Finance \& Trade Economics, 2018, 39(6): 147-161. ]

[20] 刘莉文, 张明. 国外高速铁路对区域空间经济的影响研 究及启示 [J]. 国际城市规划, 2018, 33(1): 95-100. [Liu Liwen, Zhang Ming. The impacts of high speed rail on regional and spatial economy abroad and the enlightenment to China. Urban Planning International, 2018, 33 (1): 95-100. ]

[21] 宋欣, 孙伟, 王䂞. 长三角高铁网络时空演化格局及区 域经济影响测度研究 [J]. 长江流域资源与环境, 2020, 29(2): 296-309. [Song Xin, Sun Wei, Wang Lei. Spatialtemporal evolution patterns of high-speed rail network and its impact on regional economy in the Yangtze River Delta. Resources and Environment in the Yangtze Basin, 2020, 29(2): 296-309. ]

[22] Zhang A M, Wan Y L, Yang H J. Impacts of high-speed rail on airlines, airports and regional economies: A survey of recent research [J]. Transport Policy, 2019, 81: A1-A19.

[23] 王绍博, 郭建科, 罗小龙, 等. 高速铁路对中心城市航空 客运市场的空间影响: 基于人均时间价值视角 [J]. 地 理科学进展, 2019, 38(11): 1665-1674. [Wang Shaobo, Guo Jianke, Luo Xiaolong, et al. Spatial differentiation 
of the impact of high-speed rail on aviation passenger market in central cities of China. Progress in Geography, 2019, 38(11): 1665-1674. ]

[24] 倪维秋, 廖茂林. 高速铁路对中国省会城市旅游经济联 系的空间影响 [J]. 中国人口・资源与环境, 2018, 28(3): 160-168. [Ni Weiqiu, Liao Maolin. Spatial influence of high-speed rails on the tourism economic connection of provincial cities in China. China Population, Resources and Environment, 2018, 28(3): 160-168. ]

[25] 黄泰, 席建超, 葛全胜. 高铁对长三角区域旅游一体化 影响计量研究 [J]. 长江流域资源与环境, 2017, 26(9): 1311-1322. [Huang Tai, Xi Jianchao, Ge Quansheng. Impact of high-speed rail on regional tourism integration in the Yangtze River Delta. Resources and Environment in the Yangtze Basin, 2017, 26(9): 1311-1322. ]

[26] 邵博, 李若然, 叶羽, 等. 高铁网络下可达性与区域经济 联系的空间格局演变: 基于福建省的实证分析 $[\mathrm{J}]$. 华东 经济管理, 2020, 34(8): 33-43. [Shao Bo, Li Ruoran, Ye Chong, et al. Spatial pattern evolution of accessibility and regional economic connections under high-speed railway network: Empirical analysis based on Fujian Province. East China Economic Management, 2020, 34(8): 33-43. ]

[27] 郭建科, 王绍博, 李博, 等. 哈大高铁对东北城市旅游经 济联系的空间影响 [J]. 地理科学, 2016, 36(4): 521529. [Guo Jianke, Wang Shaobo, Li Bo, et al. The spatial effect of Harbin-Dalian high-speed rail to the northeast city tourism economic link. Scientia Geographica Sinica, 2016, 36(4): 521-529. ]

[28] 孔令章, 李晓东, 白洋, 等. 长距离高铁对沿线城市旅游 经济联系的空间影响及角色分析: 以兰新高铁为例 [J]. 干旱区地理, 2019, 42(3): 681-688. [Kong Lingzhang, Li Xiaodong, Bai Yang, et al. Spatial effect of long-distance high-speed railway on tourism economic link and role analysis of cities along the railway: A case of Lanzhou-Xinjiang high-speed rail. Arid Land Geography, 2019, 42(3): 681-688. ]

[29] 鄢慧丽, 王强, 熊浩, 等. 中国 “四纵四横” 高铁对沿线站 点城市可达性及其经济联系的影响 [J]. 经济地理, 2020, 40(1): 57-67. [Yan Huili, Wang Qiang, Xiong Hao, et al. The effect of Chinese "four vertical and four horizontal" high-speed railways on the accessibility and economic relations of the cities along the line. Economic Geography, 2020, 40(1): 57-67. ]

[30] 于涛, 张京祥, 罗小龙, 等. 人本视角下的城市发展动力 与治理创新: 基于南京实证研究 [J]. 城市规划, 2018, 42 (3): 50-58. [Yu Tao, Zhang Jingxiang, Luo Xiaolong, et al. Dynamics of urban development and governance innovation from the humanistic perspective: An empirical study of Nanjing. City Planning Review, 2018, 42(3): 5058. ]

[31] 胡国建, 陆玉麒. 基于企业视角的城市网络研究进展、 思考和展望 [J]. 地理科学进展, 2020, 39(9): 1587-1596. [Hu Guojian, Lu Yuqi. Progress, thoughts, and prospect of urban network research based on enterprise perspective. Progress in Geography, 2020, 39(9): 1587- 1596. ]

[32] Pan F H, Bi W K, Lenzer J, et al. Mapping urban networks through inter- firm service relationships: The case of China [J]. Urban Studies, 2017, 54(16): 3639-3654.

[33] 李哲睿, 甄峰, 傅行行. 基于企业股权关联的城市网络 研究: 以长三角地区为例 [J]. 地理科学, 2019, 39(11): 1763-1770. [Li Zherui, Zhen Feng, Fu Xingxing. Mapping urban network through inter-firm investment relationship: A case study of Yangtze River Delta. Scientia Geographica Sinica, 2019, 39(11): 1763-1770. ]

[34] 王姣娥, 杜德林, 金凤君. 多元交通流视角下的空间级 联系统比较与地理空间约束 [J]. 地理学报, 2019, 74 (12): 2482-2494. [Wang Jiao'e, Du Delin, Jin Fengjun. Comparison of spatial structure and linkage systems and geographic constraints: A perspective of multiple traffic flows. Acta Geographica Sinica, 2019, 74(12): 2482-2494. ]

[35] 吴凤连, 郝丽莎, 王晓歌, 等. 基于高铁联系的中国东部 城市服务业发展潜力格局: 社会网络分析视角 [J]. 经 济地理, 2020, 40(4): 145-154. [Wu Fenglian, Hao Lisha, Wang Xiaoge, et al. Development potential pattern of service industry in the eastern Chinese cities based on highspeed railway connection: From the perspective of social network analysis. Economic Geography, 2020, 40(4): 145-154. ]

[36] 薛德升, 邹小华. 基于中资商业银行全球空间扩展的世 界城市网络及其影响因素 [J]. 地理学报, 2018, 73(6): 989-1001. [Xue Desheng, Zou Xiaohua. The world city network based on the global expansion of Chinese commercial banks and its influencing factors. Acta Geographica Sinica, 2018, 73(6): 989-1001. ]

[37] 冷炳荣, 杨永春, 谭一洺, 等. 结构动力机制视角下的城 市网络解释框架 [J]. 地理研究, 2013, 32(7): 12431252. [Leng Bingrong, Yang Yongchun, Tan Yiming, et al. City network studies: A conceptual explanatory framework. Geographical Research, 2013, 32(7): 1243-1252. ]

[38] 周一星. 城市地理学 [M]. 北京: 商务印书馆, 1995. [Zhou Yixing. Urban geography. Beijing, China: The Commercial Press, 1995. ]

[39] 高鹏, 何丹, 宁越敏, 等. 长江中游城市群社团结构演化 及其邻近机制: 基于生产性服务企业网络分析 [J]. 地 理科学, 2019, 39(4): 578-586. [Gao Peng, He Dan, Ning Yuemin, et al. Community structure and proximity mech- 
anism of city clusters in Middle Reach of the Yangtze River: Based on producer service firms' network. Scientia Geographica Sinica, 2019, 39(4): 578-586. ]

[40] 季菲菲, 陈雯. 长三角地区金融机构网络分布格局与扩 张机理: 以城市商业银行为例 [J]. 地理科学进展, 2014, 33(9): 1241-1251. [Ji Feifei, Chen Wen. Distribution pattern and expansion mechanism of financial insti- tution network in the Yangtze River Delta. Progress in Geography, 2014, 33(9): 1241-1251. ]

[41] 方创琳, 刘海猛, 罗奎, 等. 中国人文地理综合区划 [J]. 地理学报, 2017, 72(2): 179-196. [Fang Chuanglin, Liu Haimeng. Luo Kui, et al. Comprehensive regionalization of human geography in China. Acta Geographica Sinica, 2017, 72(2): 179-196. ]

\title{
Impact of high-speed railway network on inter-city connections and its heterogeneity: Taking Nanjing City as the center of network
}

\author{
LIU Mengyu, SHEN Lizhen* \\ (School of Architecture and Urban Planning, Nanjing University, Nanjing 210093, China)
}

\begin{abstract}
The fast-growing high-speed rail network has a deep impact on regional socioeconomic systems. This study used the external horizontal economic linkage data of the whole industry in Nanjing City from 2000 to 2019 to measure the change in the economic linkages between Nanjing and other cities. On the basis of revealing the connection patterns and changes between city-dyads, the study quantitatively identified and analyzed the heterogeneity of the impact of high-speed rail on city-dyads with different attributes. The results show that: 1) The strength of the connections between Nanjing and different cities across the country exhibits obvious hierarchical, regional distribution, and portal characteristics. 2) The difference in economic development level and the strength of the transportation connection between city-dyads are the key influencing factors of the strength of the connections. The development of high-speed rail strengthens the connections between city-dyads and to a certain extent aggravates the polarization of the geographical pattern of connections. 3) The high-speed rail shows obvious heterogeneity in the degree of impact on different types of city-dyads. City-dyads with short time distances, high degree of cooperation guided by government policies, large differences in GDP, and small differences in innovation capacity are more likely to benefit from the development of high-speed rail. According to the conclusions of this research, it is possible to intervene and guide specific cities to participate in the construction of urban networks. Under the influence of the high-speed rail, city-dyad may further directionally drive the flow of factors between cities.
\end{abstract}

Keywords: high-speed rail; city connection; city network; flow space; city-dyad; Nanjing 